\title{
Mean endpoints
}

Force field without VFB (FFnv)
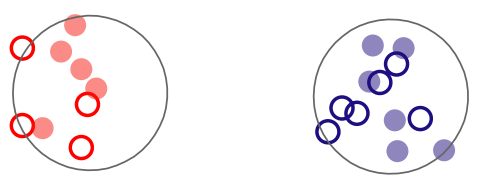

0

$\mathrm{O}$
Force field with VFB (FFv)

$$
\begin{array}{ll}
\text { O day1 early } \\
\text { day1 late } \\
\text { O day2 early } \\
\text { - day2 late }
\end{array}
$$
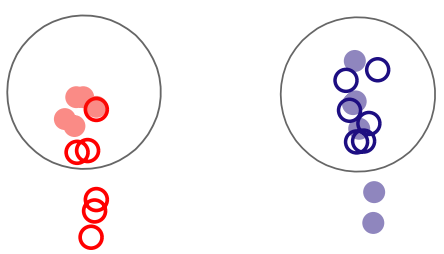

8

-

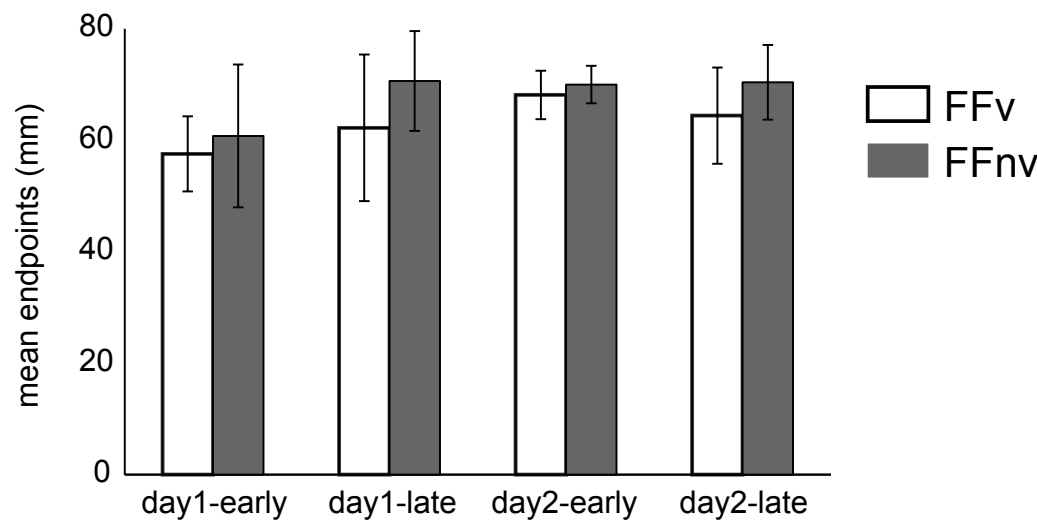

Figure S1. Mean movement endpoints of each of the subjects in force field without visual feedback (FFnv,left) and with (FFv,right). Each circle is a subject's mean final hand position across the early $(1: 40)$ and late (181:220) trials of both days. Bar plot of the mean endpoints across all subjects in each group during the early and late phases of day 1 and day 2 training. Vertical lines are \pm 1 standard error of the mean. 